Fixed Point Theory, 20(2019), No. 2, 623-634

DOI: $10.24193 /$ fpt-ro.2019.2.41

http://www.math.ubbcluj.ro/ nodeacj/sfptcj.html

\title{
FIXED POINT THEOREMS FOR MULTIVALUED NONSELF KANNAN-BERINDE CONTRACTION MAPPINGS IN COMPLETE METRIC SPACES
}

\author{
JENWIT PUANGPEE* AND SUTHEP SUANTAI** \\ *Department of Mathematics, Faculty of Science \\ Chiang Mai University, Chiang Mai 50200, Thailand \\ E-mail: jenwit.pp@hotmail.com \\ **Data Science Research Center \\ Department of Mathematics, Faculty of Science \\ Chiang Mai University, Chiang Mai 50200, Thailand \\ E-mail: suthep.s@cmu.ac.th
}

\begin{abstract}
In this paper, a new type of multivalued nonself Kannan-Berinde contraction mappings in metric spaces is introduced and studied. We establish the existence of fixed points of this type of mappings on a complete convex metric space. Our main results extend and generalize many wellknown fixed point theorems of many other authors in the literature. We also give an example to illustrate our main results.

Key Words and Phrases: Fixed point, nonself multivalued mappings, Kannan-Berinde contraction, Rothe's boundary condition.
\end{abstract}

2010 Mathematics Subject Classification: 47H10, 54H25, 47H09.

Acknowledgment. The authors would like to thank Chiang Mai University, Chiang Mai, Thailand for the financial support.

\section{REFERENCES}

[1] M.R. Alfuraidan, Fixed points of multivalued mappings in modular function spaces with a graph, Fixed Point Theory Appl., 2015(2015), no. 2015:42.

[2] M.R. Alfuraidan, Remarks on monotone multivalued mappings on a metric space with a graph, J. Inequal. Appl., 2015(2015), no. 7.

[3] M.A. Alghamdi, V. Berinde, N. Shahzad, Fixed points of multivalued nonself almost contractions, J. Appl. Math., 2013(2013)

[4] N.A. Assad, On some nonself nonlinear contractions, Math. Japon., 33(1988), no. 1, 17-26.

[5] N.A. Assad, On some nonself mappings in Banach spaces, Math. Japon., 33(1988), no. 4, 501-515.

[6] N.A. Assad, A fixed point theorem in Banach space, Publ. Inst. Math. (Beograd) (N.S.), 47(1990), no. 61, 137-140.

[7] N.A. Assad, A fixed point theorem for some non-self-mappings, Tamkang J. Math., 21(1990), no. $4,387-393$

[8] N.A. Assad, W.A. Krik, Fixed point theorems for set-valued mappings of contractive type, Pacific J. Math., 43(1972), no. 3, 553-562. 
[9] L. Balog, V. Berinde, Fixed point theorems for nonself Kannan type contractions in Banach spaces endowed with a graph, Carpathian J. Math., 32(2016), no. 3, 293-302.

[10] S. Banach, Sur les operations dans les ensembles abstraits et leur application aux equations itegrales, Fundam. Math., 3(1922), 133-181.

[11] V. Berinde, Approximating fixed points of weak contractions using the Picard iteration, Nonlinear Analysis Forum, 9(2004), no. 1, 43-53.

[12] M. Berinde, V. Berinde, On a general class multi-valued weakly Picard mappings, J. Math. Anal. Appl., 326(2007), 772-782.

[13] V. Berinde, M. Pacurar, Fixed point theorems for nonself single-valued almost contractions, Fixed Point Theory, 14(2013), no. 2, 301-312.

[14] V. Berinde, M.A. Petric, Fixed point theorems for cyclic non-self singlevalued almost contractions, Carpathian J. Math., 31(2015), no. 3, 289-296.

[15] F. Bojor, Fixed point theorems for Reich type contraction on metric spaces with a graph, Nonlinear Anal., 75(2012), 3895-3901.

[16] A. Branciari, A fixed point theorem for mappings satisfying a general contractive condition of integral type, Int. J. Math. Math. Sci., 29(2002), no. 9, 531-536.

[17] S.K. Chatterjea, Fixed point theorems, C.R. Acad. Bulgare Sci., 25(1972), 727-730.

[18] L.B. Ćirić, A remark on Rhoades' fixed point theorem for non-self mappings, Internat. J. Math. Math. Sci., 16(1993), 397-400.

[19] L.B. Ćirić, J.S. Ume, M.S. Khan, H.K. Pathak, On some nonself mappings, Math. Nachr., 251(2003), 28-33.

[20] A. Hanjing, S. Suantai, Coincidence point and fixed point theorems for a new type of Gcontraction multivalued mappings on a metric space endowed with a graph, Fixed Point Theory Appl., 2015(2015), no. 2015:171.

[21] S. Itoh, Multivalued generalized contractions and fixed point theorems, Comment. Math. Univ. Carolinae, 18(1977), 247-258.

[22] R. Kannan, Some results on fixed points, Bull. Calcutta Math. Soc., 10(1968), 71-76.

[23] W.A. Kirk, P.S. Srinivasan, P. Veeramani, Fixed points for mappings satisfying cyclical contractive conditions, Fixed Point Theory, 4(2003), no. 1, 79-89.

[24] C. Klanarong, S. Suantai, Coincidence point theorems for some multi-valued mappings in complete metric spaces endowed with a graph, Fixed Point Theory Appl., 2015(2015), no. 2015:129.

[25] S.B. Nadler, Multi-valued contraction mappings, Pac. J. Math., 30(1969), no. 2, 475-488.

[26] J.J. Nieto, R. Rodriguez-Lopez, Contractive mapping theorems in partially ordered sets and applications to ordinary differential equation, 22(2005), 223-239.

[27] J.J. Nieto, R. Rodriguez-Lopez, Existence and uniqueness of fixed point in partially ordered sets and applications to ordinary differential equation, Acta Math. Sin. (Engl. Ser.), 23(2007), no. $12,2205-2212$.

[28] A.C.M. Ran, M.C.B. Reurings, A fixed point theorem in partially ordered sets and some applications to matrix equations, Proc. Amer. Math. Soc., 132(2004), no. 5, 1435-1443.

[29] A. Roldán, J. Martinez-Moreno, C. Roldán, E. Karapinar, Some remarks on multidimensional fixed point theorems, Fixed Point Theory, 15(2014), no. 2, 545-558.

[30] P. Sridarat, S. Suantai, Caristi fixed point theorems in metric spaces with a graph and its applications, J. Nonlinear Convex Anal., 17(2016), no. 7, 1417-1428.

[31] W. Takanashi, Nonlinear Functional Analysis, Yokohama Publishers, Yokohama, 2000.

[32] J. Tiammee, S. Suantai, Coincidence point theorems for graph-preserving multi-valued mappings, Fixed Point Theory Appl., 2014(2014), no. 2014:70.

[33] K. Yanagi, A common fixed point theorem for a sequence of mutivalued mappings, Publ. Z. Res. Inst. Math. Sci., 15(1979), 47-52.

[34] T. Zamfirescu, Fixed point theorems in metric spaces, Arch. Math. (Basel), 23(1972), 292-298.

Received: March 20, 2017; Accepted: July 15, $201 \%$. 